\title{
Aerodynamically-Actuated Radical Shape-Change Concept
}

\author{
Thomas G. Ivanco, ${ }^{*}$ Marie L. Ivanco, ${ }^{\dagger}$ and Ersin Ancel ${ }^{\ddagger}$ \\ NASA Langley Research Center, Hampton, VA 23681 \\ Amanda L. Grubb ${ }^{\S}$ \\ Georgia Institute of Technology, Atlanta, GA 30332 \\ Supranamaaya Prasad ${ }^{* *}$ \\ York High School, Yorktown, VA 23692
}

\begin{abstract}
Aerodynamically-actuated radical shape change (AARSC) is a novel concept that enables flight vehicles to conduct a mission profile containing radically different flight regimes while possibly mitigating the typical penalties incurred by radical geometric change. Weight penalties are mitigated by utilizing a primary flight control to generate aerodynamic loads that then drive a shape-change actuation. The flight mission profile used to analyze the AARSC concept is that of a transport aircraft that cruises at a lower altitude than typical transports. Based upon a preliminary analysis, substantial fuel savings are realized for mission ranges below 2000 NM by comparison to a state-of-the-art baseline, with an increasing impact as mission range is reduced. The predicted savings are so significant at short-haul ranges that the shape-change concept rivals the fuel-burn performance of turboprop aircraft while completing missions in less time than typical jet aicraft. Lower-altitude cruise has also been sought after in recent years for environmental benefits, however, the performance penalty to conventional aircraft was prohibitive. AARSC may enable the opportunity to realize the environmental benefits of lower-altitude emissions coupled with mission fuel savings. The findings of this study also reveal that the AARSC concept appears to be controllable, turbulence susceptibility is likely not an issue, and the shape change concept appears to be mechanically and aerodynamically feasible.
\end{abstract}

\section{Nomenclature}

$A A R S C=$ Aerodynamically Actuated Radical Shape Change

$C A T=$ Clear Air Turbulence

$D A R P A=$ Defense Advanced Research Projects Agency

$q \quad=$ Dynamic pressure $(\mathrm{psf})$

$W_{a c t} \quad=$ Weight penalty factor for wing actuation

\section{Introduction}

$\mathrm{N}$ EARLY all aircraft utilize some form of shape change such as Fowler flaps, spoilers, and leading edge slats. The degree to which these shape change devices alter the aircraft is dictated by the degree to which the flight regime changes throughout a mission profile. During more extreme changes in flight regime, such as the long-duration loiter and high-speed dash requirements of the recent Defense Advanced Research Projects Agency (DARPA) morphing program, more extreme shape change is required to enable the desired capabilities. ${ }^{1}$ Other examples of substantial shape change include variable sweep observed in production aircraft such as the F-111, F-14, B-1, MiG-23, and Mirage $\mathrm{G}$ and telescoping wings employed in the FS-29 competition glider. The enabling technologies for these shape-change concepts often face difficult challenges such as weight penalties, complexity of design, the need for large acreage

\footnotetext{
* Research Aerospace Engineer, Aeroelasticity Branch, MS 340, AIAA senior member.

† Aerospace Engineer, Space Mission Analysis Branch, MS 462, AIAA member.

* Aerospace Engineer, Aeronautics Systems Analysis Branch, MS 442, AIAA member.

$\S$ NASA Intern, PhD Student, School of Aerospace Engineering, AIAA member.

${ }^{* *}$ NASA Volunteer, Student, York High School.
} 
flexible skin, and a difficulty in maintaining a clean outer mold line (OML) for transonic/supersonic operation. ${ }^{1-3}$ These challenges increase the maintenance requirements and cost and threaten performance and reliability. As a result, radical shape change is rarely beneficial to implement.

A team of NASA engineers developed a novel system concept, which addresses the typical drawbacks of radical shape change by using aerodynamic loads rather than mechanical actuation. The flight mission profile used to evaluate this Aerodynamically-Actuated Radical Shape Change (AARSC) concept is that of a transport aircraft that cruises at a lower altitude. The AARSC concept proposes to cruise in the vicinity of 15,000 to 25,000 feet to save fuel and reduce environmental impact. The increase in dynamic pressure realized at this lower cruise altitude warrants a radical change in the lifting surface of the aircraft in order to enable typical take-off and landing speeds without sacrificing cruise performance. AARSC offers a radical shape-change capability with tolerable weight penalty and complexity.

This paper will begin with lessons learned from the DARPA morphing program and will then introduce the design concept that leverages these lessons learned. Several challenge areas were identified by the team and include: aerodynamic performance, structural weight, the actuation mechanism, aircraft controllability, susceptibility to turbulence, engine integration, and mission fuel-burn analyses. A systematic evaluation of the AARSC concept as applied to a lower-altitude transport mission will be presented with respect to each design challenge based upon the results of a preliminary study. To serve as a reference, the same analyses were applied to a Boeing 737-800 in order to represent the current state-of-the-art for a middle of the market single-aisle aircraft.

\section{Lessons Learned from Previous Shape-Change Efforts}

The DARPA morphing program investigated radical shape-change concepts with several innovative ideas such as independently-variable sweep and area, folding wings, and other novel concepts. ${ }^{2,3}$ This morphing program was studied in order to leverage the lessons learned for application to the AARSC concept.

Structural weight required by strong actuators and drive-train systems was an obvious penalty to morphing aircraft. However, a less obvious penalty was the weight required by the connecting structures to absorb and distribute the point-loads created by these actuators. The combined weight of motors/actuators, drive-shafts/linkages, mounting brackets and connections to typical bulkheads far exceeded the weight of the moving aerodynamic surfaces. This realization is what inspired the AARSC concept, which instead uses aerodynamic loads generated by the moving surfaces to serve as the actuating mechanism.

Another significant challenge experienced during the morphing program was the requirement for large acreage flexible skin. While great advances were indeed made in material development, the weight and performance penalty of these flexible skins were still prohibitive. Additionally, the lifecycle of the flexible skins was nearly insufficient for a relatively short wind-tunnel program and would unlikely be a practical solution for production use without a substantial technology leap. ${ }^{1}$ Therefore, the AARSC team sought to incorporate a shape-change concept that did not require a large amount of flexible skin.

Finally, a significant challenge for one of the folding-wing morphing concepts was creating a clean OML for transonic/supersonic flight. The area rule dictates where the fuselage body should have minimum cross-sectional area, typically at the wing root location. However, the folding wing adds area to the fuselage in this region. Furthermore, gaps between the wing surface and fuselage increased wave drag. Smoothing these gaps would require either altering the upper wing surface or the fuselage interface. Either option would then impact low-speed performance. Therefore, a shape-change concept that offered a clean cruise OML was sought by the AARSC team.

\section{Evaluation of the AARSC Concept and the Design Challenges}

Leveraging the lessons learned from previous shape-change efforts, the AARSC team designed a telescoping wing concept, as shown in Fig. 1, actuated by active winglets. The concept includes upper and lower surface winglets that are independently controlled to enable redundancy and the ability to control roll independently of wing extension and retraction. Additionally, the multiple winglets enable the ability to tailor the tip vortex for reduced drag, or to operate opposed to increase drag on approach.

If the active winglets are used for roll-control in normal flight, in lieu of ailerons, the requirement for additional actuators and linkages is reduced in addition to avoiding aeroelastic reversal effects. Furthermore, if pivoted about the center of pressure, the actuator strength required to operate the winglets would be smaller than those required for ailerons.

With the telescoping portion of the wing fitting entirely into the wing root, the requirement for large acreage flexible skin is eliminated and the cruise OML remains clean and optimized for transonic performance. The 
telescoping portion is only exposed during slow speeds, and can be preslotted, enhanced with vortex generators, and utilize an airfoil optimized for subsonic high lift.

Cruising at lower altitudes offers some performance benefits including an increase in the speed of sound. Therefore, a vehicle traveling at the same speed as the baseline will have a lower Mach number resulting in less wave drag and/or less wing-sweep requirements. Less wing sweep increases the aerodynamic efficiency and reduces the structural weight. Additionally, there is less pressure differential on the fuselage. For example, the baseline vehicle is designed for a maximum altitude of 41,000 feet while maintaining an internal pressure equivalent to 8,000 feet. The AARSC concept cruising at 20,000 feet will have half of the pressure differential, enabling the use of an elliptical cross-section and significantly reducing the HVAC pressure requirements. This elliptical cross-section results in a significantly lower cross-sectional area and can mitigate the drag rise on the fuselage due to the increased density at lower altitude. The under-floor storage lost with an elliptical concept can be moved behind the passenger cabin. To maintain the same storage volume as the baseline, the AARSC fuselage is required to be 10 feet longer, and it is envisioned that the engines would be fuselage-mounted in this area behind the passenger cabin. This increase in length would make the AARSC concept comparable to a 737-900 length with the same payload as a 737-800. Therefore, all families of 737 aircraft (with the exception of the 900) could be replicated with the AARSC concept while utilizing comparable-length landing gear. A sketch of the AARSC fuselage by comparison to the baseline is depicted in Fig. 2.

In addition to potential performance benefits, cruising at lower altitude offers significant environmental benefits. ${ }^{4-8}$ At high-altitude, NOx emissions have a greater environmental impact, particularly when emitted at or above the tropopause. ${ }^{7}$ Additionally, the production of contrails further increase the radiative forcing that drives global warming. ${ }^{8}$ Therefore, a reduction to the environmental impact of aviation emissions can be realized with lower-altitude cruise, even if the magnitude of the emissions remain unchanged.

The AARSC concept can also be applied to different mission scenarios such as a loiter-dash vehicle, and it can be applied to different shapechange options such as a folding wing. Exploring all feasible uses of the AARSC concept is beyond the scope of the present investigation. Therefore, AARSC in this document will refer to the telescoping transport wing concept described above.

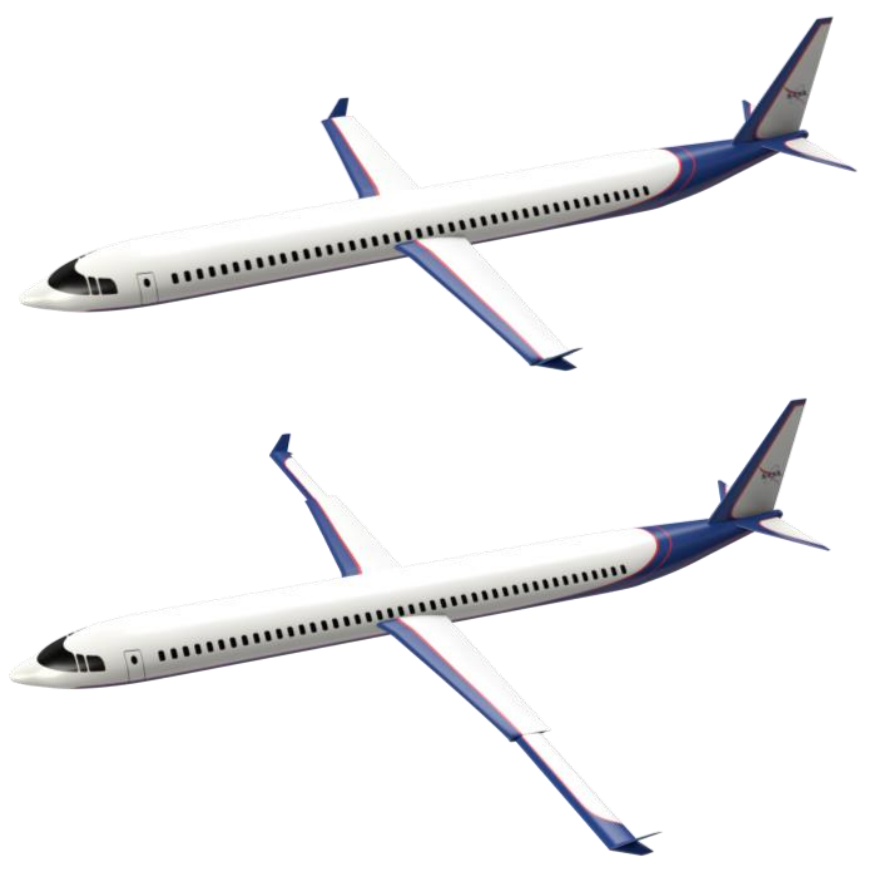

Figure 1. AARSC concept, telescoping wing with active winglets. 


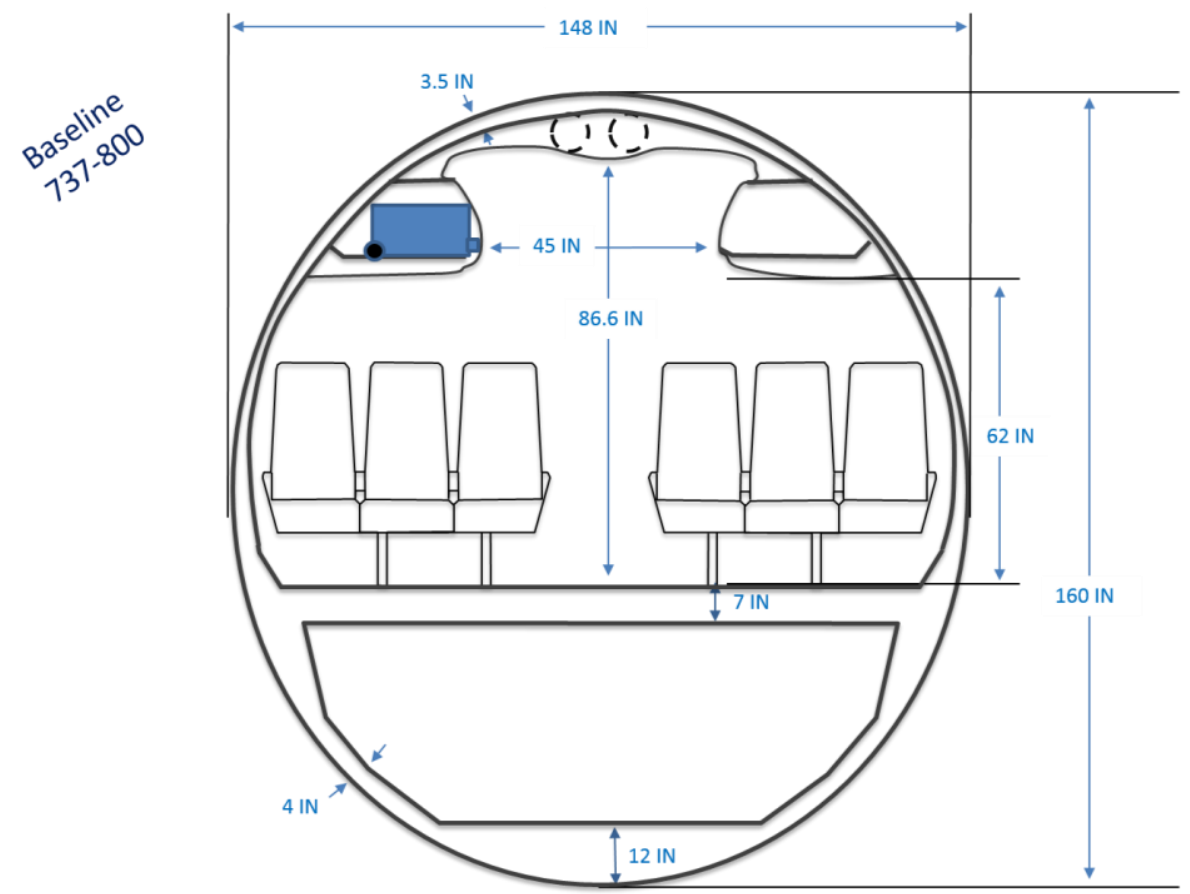

\section{a.) $737-800$ baseline}

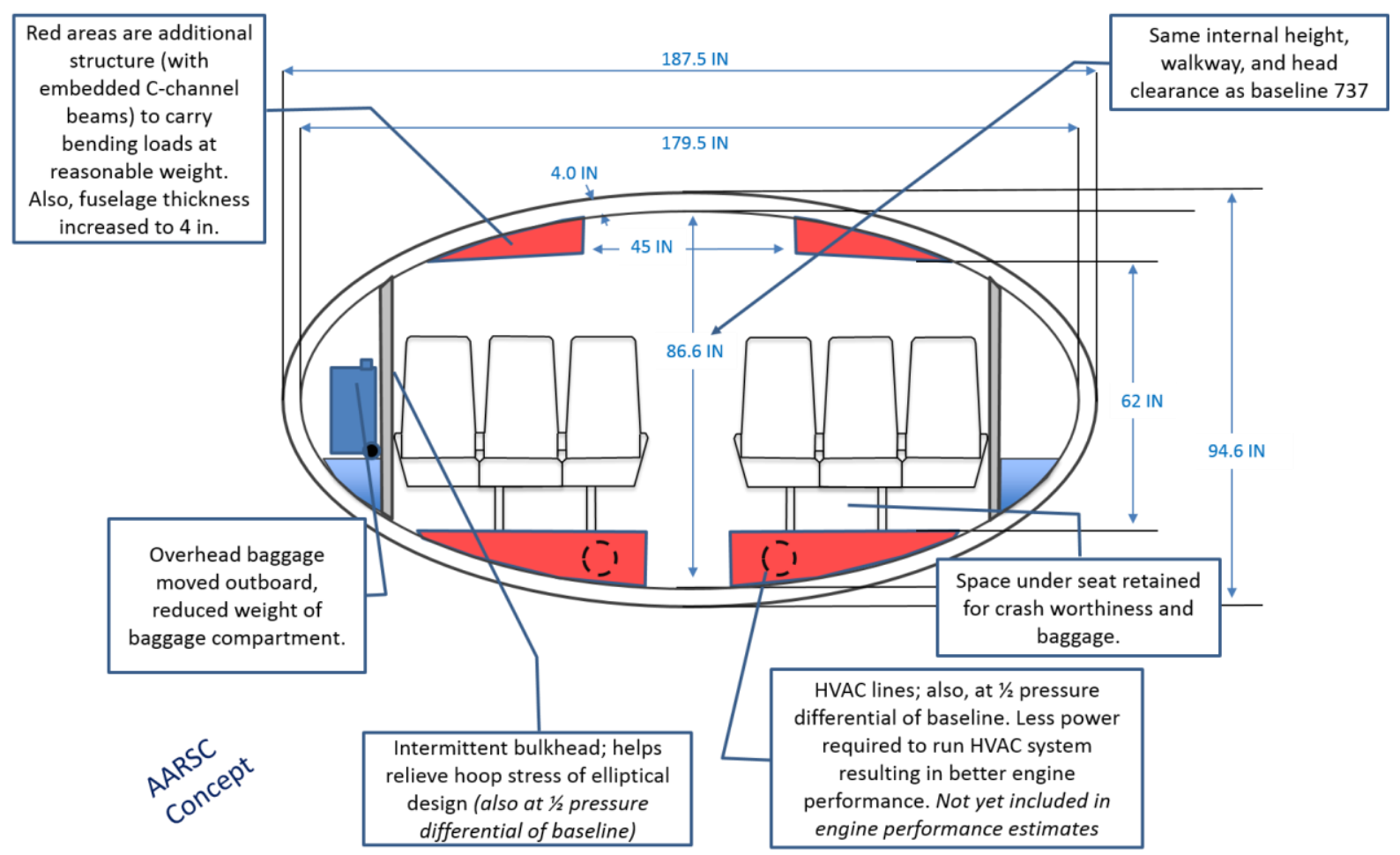

b.) AARSC concept

Figure 2. Sketch of fuselage cross-sections.

American Institute of Aeronautics and Astronautics 


\section{A. Aerodynamic Performance}

Aerodynamic performance of the AARSC concept was estimated using a combination of empirical relations from Ref. 9, finite wing theory from Refs. 10 and 11, and an unstructured-grid Navier-Stokes CFD code, FUN3D. ${ }^{12}$ Empirical relations and basic wing theory were used to estimate the performance of the wing and tail surfaces in the cruise configuration, and CFD was used to estimate the performance of the fuselage. The cruise wing and tail surfaces are similar to conventional designs and should adequately follow the trends of empirical relations. Additionally, detailed design of the lifting surfaces in a CFD study is not trivial and beyond the resource limitations of the present study. An elliptical fuselage, however, is uncharacteristic of typical transports and it is doubtful that empirical relations will adequately capture the performance. Furthermore, the fuselage is not a lifting surface in cruise and is not subject to complex twist distributions or thickness optimization simplifying CFD analysis. Therefore, CFD results were used to quantify the performance of the elliptical fuselage at various altitudes.

\section{Cruise Wing}

Considering the cruise wing, if one were to scale the planform area of the baseline wing by the same ratio as the increase in dynamic pressure $(q)$ experienced by the AARSC concept, the wing performance (lift to drag ratio) should be very similar. However, due to the increase in temperature and therefore the speed of sound at lower altitude, the AARSC concept operates at a lower Mach number ( 0.73 vs. 0.78$)$ to achieve the same cruise speed. Flying at a lower Mach number allows the wing to be reduced in sweep and slightly reduced in area beyond that required to match the ratio in $q$. This additional decrease in area required to produce the same lift will slightly reduce the drag and improve vehicle performance. Furthermore, the AARSC concept utilizes upper and lower surface winglets that can also be tailored to minimize induced drag. This was approximated in the empirical relations by increasing the Oswald efficiency factor of a finite wing to simulate the effect of the dual winglets. This also results in a slight efficiency improvement for the AARSC concept. Both of these items were offset by a slight reduction in the aspect ratio and an increase in the thickness to chord ratio. The thickness to chord ratio increase was chosen to maintain comparable wing thickness with the reduced-chord AARSC wing as the baseline aircraft. At a lower Mach number, this thickness increase incurs less penalty than the baseline configuration otherwise would. Both of these geometric changes facilitate the shape-change concept, and help minimize weight penalty. The cumulative effect of these factors is that the AARSC wing has a slightly improved performance realizing approximately six percent less wing drag than the baseline to produce the same lift. This results in approximately a 2.9 percent reduction of aircraft drag.

\section{Vertical Tail}

Vertical tail performance calculations assumed that the AARSC concept would utilize fuselage-mounted engines enabling a 15 percent reduction in vertical-tail area due to a substantial reduction in engine-out yaw characteristics. It was also assumed that the tail moment arm would be the same as the baseline and that the aft C.G. shift of the fuselagemounted engines would be offset by the extension of the fuselage length to accommodate checked-baggage. Similar sweep and area reduction calculations were also done as that for the wing, and the drag rise due to increased dynamic pressure was somewhat mitigated. The cumulative effect is a 33 percent increase in vertical tail drag resulting in a 1.3 percent increase in aircraft drag.

To simplify comparisons with the baseline aircraft, a conventional tail configuration was assumed. It is acknowledged that fuselage-mounted engines are likely to result in a T-tail design, however that is not a requirement as demonstrated by the Cessna Citatation II or the Falcon 50. The weight and performance tradeoffs involved in a detailed engine intergration and tail configuration study is beyond the scope of the present paper.

\section{Horizontal Tail}

Horizontal tail performance was estimated in a very similar fashion to the vertical tail with regard to sweep and area estimation. No additional area was reduced from the horizontal tail with respect to the baseline even though the cruise wing area is substantially reduced. The rationale is that the approach wing area of the AARSC concept is comparable to the baseline and the assumption is made that the horizontal tail and elevator area are approach-critical and not cruise-critical. An additional assumption was made that active controls would be required to operate the AARSC concept, which impacts horizontal tail performance. While the use of active controls may pose a challenge for certification, they can also be employed to reduce the static margin of the aircraft. Static margin reduction was simulated by decreasing the induced drag coefficient of the horizontal tail to 30 percent of the baseline while maintaining the same level of parasite drag coefficient. This results in approximately a 22 percent reduction of horizontal tail drag resulting in a 3.98 percent reduction of aircraft drag. 


\section{Fuselage}

Moving a fixed fuselage volume through denser air results in a significant drag increase. Using the elliptical design discussed in the previous section helps to mitigate the drag increase. An image of the pressure profiles from CFD results is shown in Fig. 3 for the baseline and AARSC concept. As a result of the fuselage shape, the drag coefficient of the AARSC concept is lower than the baseline. However, due to the increase in dynamic pressure experienced by the AARSC concept, the fuselage drag is increased by almost 40 percent. This results in approximately a 12 percent increase in aircraft drag. It is likely that fuselage drag could be further reduced if a geometric optimization study was conducted.

\section{B. Structural Weight}

Detailed structural design, load computations, and resulting weight estimates are beyond the scope of the present study. Therefore, empirical relations ${ }^{9}$ were used to estimate aircraft component weights of both the baseline aircraft and the AARSC concept. These empirical relations characterize the effects of engine location, wing and tail sweep angles, thickness to chord ratios, cruise Mach numbers, maximum g-loading, control-surface area, fuselage dimensions, and HVAC requirements among other parameters. Weight saving features of the AARSC concept include a reduction in sweep of all aerodynamic surfaces, a reduction in tail area, an increase in thickness to chord ratio of the wing, a decrease in the operational Mach number of the extended wing, and a decrease in the

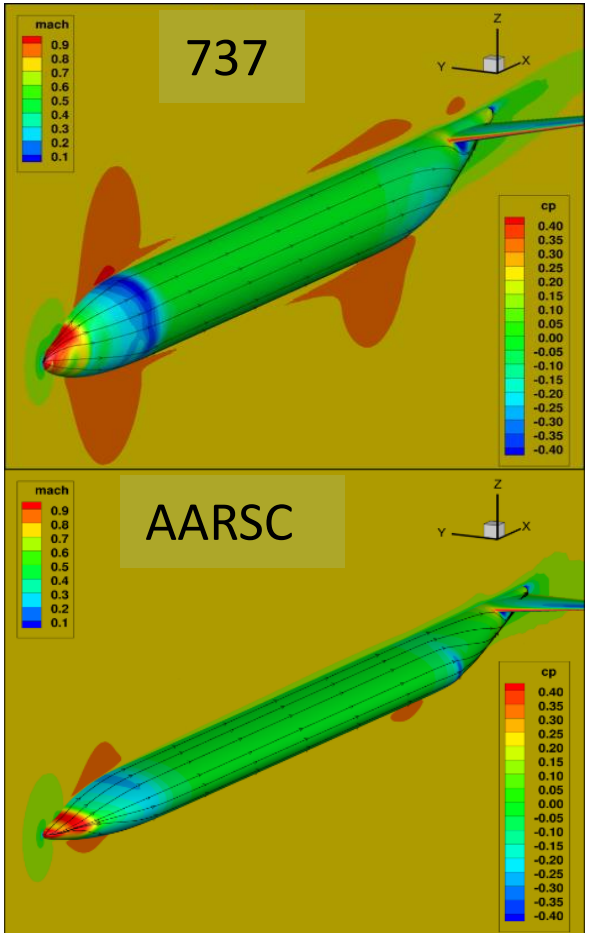

Figure 3. Pressure Profiles from CFD Results, Baseline and AARSC. design load factor of the extended wing. Recall that the wing is only extended to increase maximum lift during slow speeds and will not be extended during high-speed flight. Using a typical velocity to load (or "V-n" diagram) during low-speed flight, the wing will stall prior to reaching the $2.5 \mathrm{~g}$ maneuver requirement of a cruise configuration. Conversely, the estimated weight of the cruise wing subject to a $2.5 \mathrm{~g}$ load is substantially less than the extended wing.

Characterizing the weight associated with wing actuation, however, is beyond the capability of empirical relations. Therefore, the weight of the fully-extended and nonactuated wing was estimated with empirical relations, and then a variable increase factor $W_{\text {act }}$ was defined to quantify actuation weight penalty. Using a constraint that the AARSC concept and the baseline vehicle have the same gross weight results in an increase factor $W_{\text {act }}$ equal to 1.9. Therefore, the AARSC wing could be nearly double the weight of a conventional wing of comparable (extended) area, sweep, and operational requirements in order for both vehicles to have the same gross weight.

\section{Actuation Mechanism}

Figure 4 is an end-view image of the AARSC wing tip highlighting the different airfoil shapes optimized for highspeed cruise and low-speed high-lift takeoff and landing. Also shown in Fig. 4 are the conceptual locations for inboard and outboard wing spars. Weight penalty can be mitigated if the spar caps of the extendable wing are also used as the bearing surface. Not illustrated in Fig. 4 is a second row of inboard bearings. Due to the low-speed airfoil shape, these bearing surfaces offer resistance to both inplane and out-of plane bending. As the inboard wing progresses further inboard, the taper of the inboard wing enables additional clearance beyond that illustrated in Fig. 4, and will likely enable wing actuation without binding. The low-speed airfoil section can be preslotted and can contain vortex

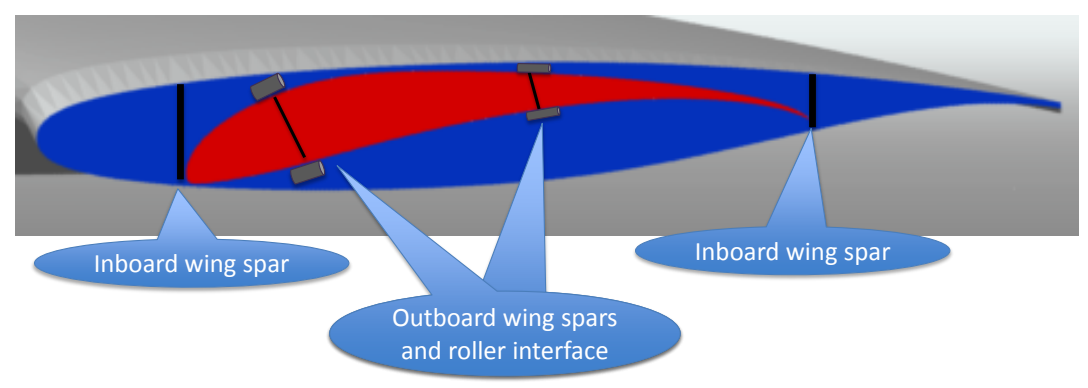

Figure 4. End-view of AARSC wing tip showing interface between outercruise wing, and inner high-lift extendable wing. 
generators on the upper surface to further enhance lift without penalty to cruise performance. It is envisioned that simplified slotted flaps and leading-edge slats will be employed on the inboard wing. The fuselage of the AARSC concept can also produce lift during low-speed flight and augment the high lift capability of the extended wing. Estimates of required wing area increase for approach speeds were then determined by relations in Refs. 5-7. Initial estimates indicate that the required outboard wing extension can fit inside of the inboard cruise wing.

Wing actuation is operated by canted winglets that extend both upward and downward from the wing tip, similar in shape to the winglets of a 737-Max. These winglets are attached to the outboard wing and are pivoted near the aerodynamic center with hydraulic actuators that are also part of a primary flight control. The wing position is then locked in either the extended or retracted positions. With dual winglets, control redundancy exists in the event of a single winglet failure. Additionally, wing actuation and roll control can be independently commanded. Finally, with dual winglets, the interaction with the tip vortex can be tailored for optimal performance at many flight regimes, or the winglets can be operated in opposition to each other to increase drag on approach as previously mentioned.

The tip of the outboard wing includes a fairing that closes the air-gap between the inboard and outboard wing sections when retracted in the cruise configuration. This feature enables a clean outer-mold line enabling adequate transonic cruise performance.

\section{Aircraft Controllability}

Controllability of the AARSC concept was investigated by developing a dynamic model of both the baseline and AARSC concept in MATLAB Simulink. Control responsiveness was evaluated for a 1-g cruise condition, and an approach condition. Control doublets were input and aircraft response in pitch, roll, and yaw were evaluated. Aerodynamic loads from the control surface deflections were estimated by using the relations in Ref. 6 . Figure 5 contains the results of a roll response of the baseline and AARSC concept to a 10 degree aileron or winglet doublet. For this condition, the AARSC concept has a much faster and more pronounced roll response. A similar study was conducted for the approach configuration of both aircraft and the AARSC concept had a slightly slower response by comparison to the baseline. Therefore, the AARSC concept may need to be augmented with spoilers for approach roll control similar to the baseline.

Work is ongoing to investigate the most efficient gain schedule for the active winglets to enable a coordinated turn without rudder input to optimize handling and efficiency.

\section{E. Susceptibility to Turbulence}

A companion paper documents the methodology and results of an investigation of atmospheric turbulence as a function of altitude and the projected sensitivity of the AARSC shape-change concept. ${ }^{13}$ This study concluded that the probability of moderate to severe turbulence encounters is lower at cruise altitudes of 20,000 feet by comparison to typical cruise altitudes of 35,000 feet. Furthermore, the response of the shape change concept to a unit gust is several factors less than the response of conventional aircraft, such as the DC-6, at the same altitude due to design parameters that include wing loading, cruise velocity, and gross weight. Therefore, it is likely that the ride quality of the AARSC concept operating at 20,000 feet is better than the baseline for typical weather conditions.

Turbulence-related airplane accident reports from the National Transportation and Safety Board were also investigated to understand if an excess of accidents occur in a particular altitude interval. The aircraft in the accident reports included small turbofan, large turbofan, and large turboprop aircraft. Work is underway to normalize the results by traffic density for various altitude levels.

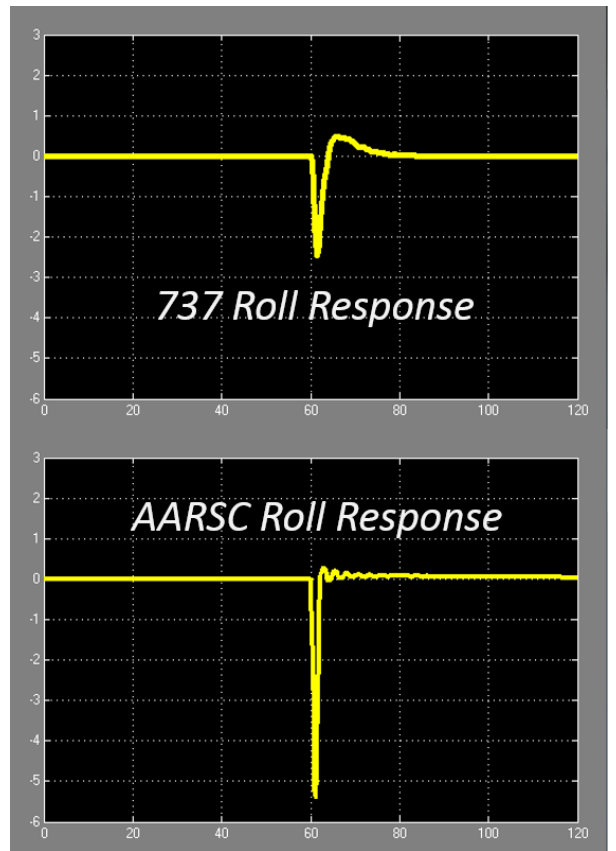

Figure 5. Roll Response of 737 Baseline and AARSC concept to control doublet.

\section{F. Engine Integration}

All fuel-burn values presented in this paper were computed using the same engine as that used in the baseline comparison, a CFM-56. The thrust specific fuel consumption of the CFM-56 at 20,000 feet and the throttle setting 
required for the AARSC concept is only slightly higher than that required for the baseline at its cruise condition. Therefore, it may indeed be a viable option to simply use the same engine as the baseline. These engine penalties are included in the analyses discussed in this paper. Proposed work will estimate the performance benefits to be gained by optimizing a turbofan engine for operation at 20,000 feet that will likely reduce the fuel burn estimates of the AARSC concept even further than those shown in the present study. Additionally, if more engines are used such that the AARSC concept is top-of-climb limited in engine performance, then the AARSC concept could also capitalize on reduced engine weight since available engine thrust is much greater at 20,000 feet than 35,000 feet.

Open-rotor and other hybrid engine concepts are currently being considered for application to the AARSC concept. These concepts may be more beneficial for AARSC than a conventional application since the AARSC concept cruises at a lower Mach number and in denser air. Leveraging these engine advancements with the AARSC profile could substantially reduce the fuel burn of the AARSC concept further below those values estimated in this paper.

\section{G. Mission Fuel-Burn Analysis}

Combining all of the performance computations, the total cruise drag of the AARSC concept is estimated to be approximately 6.5 percent higher than the baseline 737 . Conventional wisdom would dictate that an increase in cruise drag would result in an increase in mission fuel burn. However, to understand the fuel savings of the AARSC concept, one must first consider fuel burn in a typical transport flight profile.

For long range missions or aircraft "design missions," the vast majority of fuel is burned at cruise. However, for shorter range or "economic" missions, the cruise fuel burn could be as little as 22 percent. A study conducted by Boeing investigated the fuel burn of a 737-800 during various stages of a $500 \mathrm{NM}$ mission. ${ }^{14}$ Those data are represented in the $500 \mathrm{NM}$ bar of the chart shown in Fig. 6, where each color bar segment represents the percentage of the total mission fuel burned for each phase of flight. The time spent at cruise was derived for various other mission ranges to derive similar bar charts for other mission ranges, and those results are also included in Fig. 6. In this image, the "takeoff" phase refers to the maxpower takeoff and initial climb to approximately 3,000 feet. The "departure" phase is the reduced power low-speed climb to at least 10,000 feet. The "climb" phase represents the highspeed climb initiated above 10,000 feet, (and sometimes as late as 17,000 feet) and continues to the cruise altitude of approximately 35,000 feet. For the AARSC concept, the climb phase is almost entirely eliminated. Additionally, the AARSC concept is optimized for higher density air than the baseline concept. Therefore, its lowaltitude efficiency during the departure and descent phases is increased. As a result, the AARSC concept can realize

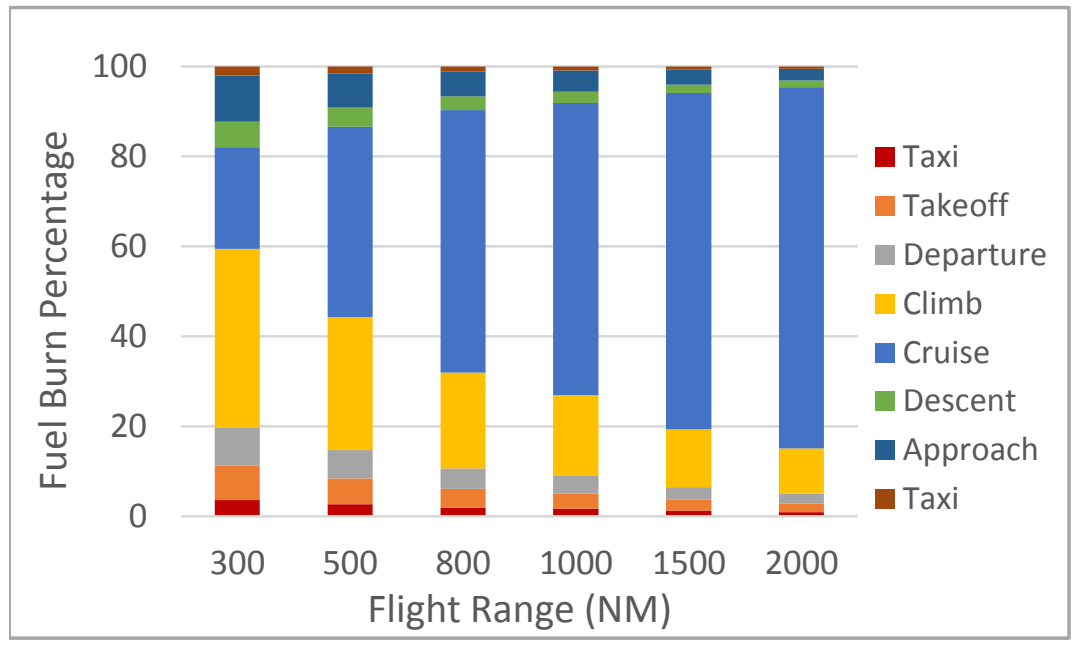

Figure 6. Fuel-burn Analysis of Baseline 737-800 by Phase of Flight. a significant mission fuel savings even though the cruise burn-rate is higher. Another small benefit of the AARSC concept is that it will enter the cruise phase sooner and will complete a given mission range in a shorter period of time than the baseline.

An analysis was performed comparing the total mission fuel burn of the baseline to the AARSC concept for several mission ranges using the empirical, textbook, and CFD results previously discussed. The results are compiled in Fig. 7. Also included in this chart are AARSC concepts designed for cruise altitudes of 15,000 and 25,000 feet in addition to the nominal 20,000 feet AARSC concept. The 15,000 feet concept very quickly becomes disadvantageous with range. The 25,000 feet concept performs better over most mission ranges, however, the realized pressure differential at higher altitude may prevent the elliptical fuselage concept. Therefore, the nominal 20,000 feet design was targeted for the majority of analyses in the present study.

For mission profiles in the 300 to $700 \mathrm{NM}$ range, the AARSC concept is predicted to rival turboprop efficiency, but will deliver passengers/cargo in less time than conventional jet aircraft. This regional and short-haul market is also where the largest gap currently exists in US-made airframe use. ${ }^{15}$ To emphasize the significance of the AARSC concept, operational data for all commercial aircraft within the National Airspace System (NAS) for one day was 
obtained from the Federal Aviation Administration. ${ }^{16}$ The operational data includes close to 20,000 domestic flights and each flight entry contains aircraft type, cruise altitude, cruise speed, great circle distance, aircraft weight classification, and the number of passengers. Analysis of this data reveals that 67 percent of these flights were below 750 NM. The 737 family (when omitting the long-range 737-800 and 737-900 series) contribute to about 17 percent of these flights, with an average range of 693 NM. For comparison, larger capacity regional aircraft such as the Embraer E-jet family and the Bombardier CRJ 700/900/1000 family contribute to approximately 14 percent of the traffic and have an average range of $570 \mathrm{NM}$.

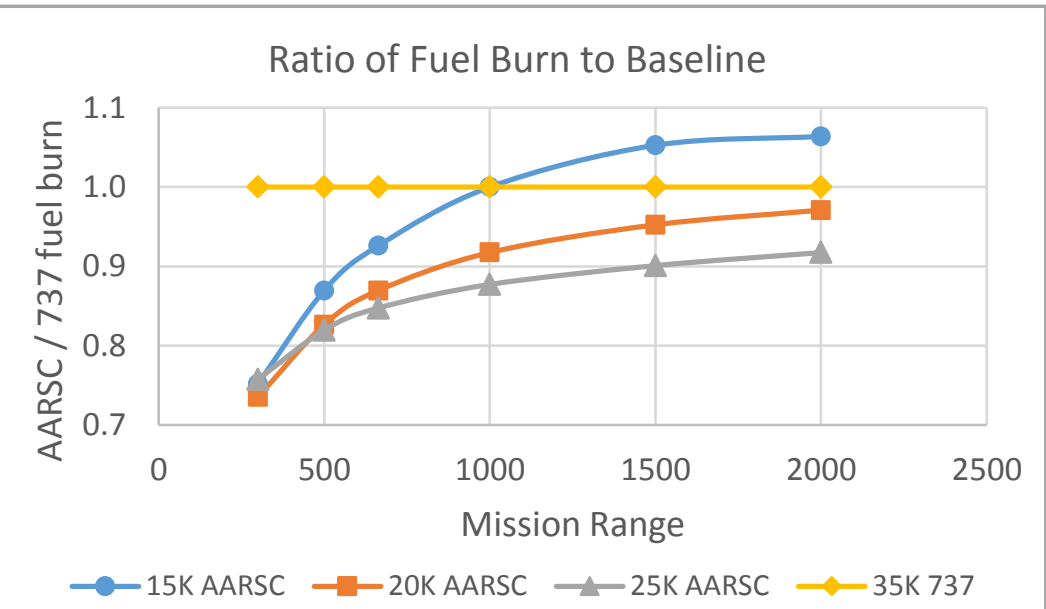

Figure 7. Mission Fuel-burn Analysis, ratio of baseline to AARSC concept; non-optimized conventional engine.

Therefore, the majority of aircraft operating within the NAS have mission ranges where the AARSC concept can realize significant fuel savings.

\section{Concluding Remarks}

AARSC is a novel concept that enables flight vehicles to conduct a mission profile containing radically different flight regimes while possibly mitigating the typical penalties incurred by radical geometric change. Weight penalties are mitigated by utilizing a primary flight control to generate aerodynamic loads that then drive a shape-change actuation. The flight mission profile used to evaluate the concept is that of a transport aircraft that cruises at a lower altitude. The flight regime change that warrants radical shape-change is the substantial difference in dynamic pressure from cruise conditions to takeoff and landing conditions. Based upon this preliminary analysis, substantial fuel savings are predicted for mission ranges below $2000 \mathrm{NM}$ by comparison to a state-of-the-art baseline, with increasing impact as mission range is reduced. On-going engine integration work is likely to further improve the performance estimates of the AARSC concept beyond those presented here. The savings may be so significant at short-haul ranges that the AARSC shape-change concept rivals the fuel-burn performance of turboprop aircraft while completing missions in less time than typical jet aircraft. This regional and short-haul market is where the largest gap exists in US-made airframe use, and the shape-change concept could revitalize this industry. More detailed structural, aerodynamic, and systems-level optimization analyses would be required to refine the preliminary mission performance predictions presented in this paper.

Lower-altitude cruise for transport aircraft is also being sought after for environmental benefits. Typical transport aircraft realize prohibitively increased fuel burn and economic impact when cruising at lower altitude and operators do not currently entertain operating below the tropopause to realize the reduction in global warming impact. The AARSC concept could enable environmentally friendlier lower-altitude flight coupled with a fuel savings.

The results of this study also revealed that the AARSC concept appears to be controllable, turbulence susceptibility is likely not an issue, and the shape change concept appears to be mechanically and aerodynamically feasible.

AARSC could also be an enabler for many other mission profiles that require radical flight regime changes such as loiter-dash concepts. Evaluating the present concept for other mission profiles is beyond the scope of this paper.

\section{References}

${ }^{1}$ Ivanco, T. G.; Scott, R. C.; Love, M. H.; Zink, S.; and Weisshaar, T. A., "Validation of the Lockheed Martin Morphing Concept with Wind Tunnel Testing," AIAA Structural Dynamics and Materials Conference, Honolulu, HI, AIAA 2007-2235, April 2007.

${ }^{2}$ Love, M. H; Zink, S.; Bye, D. R.; Rizk, S. N.; Stroud, R. L.; and White, D. W., "Demonstration of Morphing Technology through Ground and Wind Tunnel Tests," AIAA Structural Dynamics and Materials Conference, Honolulu, HI, AIAA 2007-1729, April 2007. 
${ }^{3}$ Weisshaar, T. A.; "Morphing Aircraft Systems: Historical Perspectives and Future Challenges," Journal of Aircraft, Vol. 50, No. 2, 2013, pp. 337-353

${ }^{4}$ Penner, J. E.; Lister, D. H.; Griggs, D. J.; Dokken, D. J.; and McFarland, M.; "Special Report on Aviation and the Global Atmosphere;" Intergovernmental Panel on Climate Change, Cambridge University Press, Cambridge, UK; July 2004.

${ }^{5}$ Waitz, I.; Townsend, J.; Cutcher-Gershenfeld, J.; Greitzer, E.; and Kerrebrock, J.; “Aviation and the Environment,” Report to the United States Congress; Massachusetts Institute of Technology; December 2004.

${ }^{6}$ Mahashabde, A.; Wolfe, P.; Ashok, A.; Dorbian, C.; He, Q.; Fan, A.; Lukachko, S.; Mozdzanowska, A.; Wollersheim, C.; Barrett, S. R. H.; Locke, M.; Waitz, I. A.; "Assessing the Environmental Impacts of Aircraft Noise and Emissions," Elsevier, Progress in Aerospace Sciences; 2011.

${ }^{7}$ Gauss, M.; Isaken, I. S. A.; Lee, D. S.; Sovde, O. A.; "Impact of Aircraft NOx Emissions on the Atmosphere - Tradeoffs to Reduce Impact," European Geosciences Union, Atmospheric Chemistry and Physics; Licensed under a Creative Commons License, May 2006

${ }^{8}$ Jardine, C. N.; "Calculating the Environmental Impact of Aviation Emissions," Environmental Change Institute, Oxford University Centre for the Environment; Oxford, UK; June 2005

${ }^{9}$ Raymer, Daniel P.; Aircraft Design: A Conceptual Approach; American Institute of Aeronautics and Astronautics Inc., Education Series, Washington D. C., 1992.

${ }^{10}$ McCormick, B. W.; Aerodynamics Aeronautics and Flight Mechanics; John Wiley \& Sons Inc.; New York, NY; 1995.

${ }^{11}$ Anderson, J. D. Jr.; Introduction to Flight, Fourth edition; The McGraw-Hill Companies, Inc.; New York, NY; 2000.

${ }^{12}$ FUN3D.larc.nasa.gov

${ }^{13}$ Prasad, S.; Ivanco, M. L.; Ivanco, T. G.; Ancel, E; "Characterization of Atmospheric Turbulence as a Function of Altitude," $9^{\text {th }}$ AIAA Atmospheric and Space Environments Conference, Denver CO, June 2017.

${ }^{14}$ Irrang, M. E.; "A Look at the State of Airline Fuel Conservation;" Boeing Commercial Airplanes; AGIFORS Operations Control Conference, May 2011.

${ }^{15}$ Curtis, T.; Rhoades, D. L.; Waguespack, B. P. Jr.; "Regional Jet Aircraft Competitiveness: Challenges and Opportunities;" Embry-Riddle Aeronautical University, Scholarly Commons Citation, 2013.

${ }^{16}$ Federal Aviation Administration, Advisory Circular AC 150/5300-13A; Accessed October 12, 2016. 